\title{
Some New Twists to Problems Involving the Gaussian Probability Integral
}

\author{
Marvin K. Simon, Fellow, IEEE, and Dariush Divsalar, Fellow, IEEE
}

\begin{abstract}
Using an alternate form of the Gaussian probability integral discovered a number of years ago, it is shown that the solution to a number of previously considered communication problems can be simplified and, in some cases, made more accurate (i.e., exact rather than bounded). These problems include the evaluation of: 1) bit-error probability of uncoded phaseshift keying (PSK) with Costas loop tracking; 2) word-error probability of antipodal modulation in the presence of fading; 3) bit-error probability of coded $M$-ary PSK (MPSK) over the memoryless fading channel with given channel-state information; 4) conditional symbol-error probability of MPSK in the presence of carrier synchronization error; and 5) average error probability for the binary additive white Gaussian noise (AWGN) intersymbol interference channel. Also obtained is a generalization of this new alternate form to the case of a two-dimensional Gaussian probability integral with arbitrary correlation which can be used to evaluate the symbol-error probability of MPSK with $I-Q$ unbalance.
\end{abstract}

Index Terms-Communication theory.

\section{INTRODUCTION}

A NUMBER of years ago, Craig [1] cleverly showed that the evaluation of average probability of error for the twodimensional additive white Gaussian noise (AWGN) channel could be considerably simplified by choosing the origin of coordinates for each decision region as that defined by the signal vector, as opposed to using a fixed coordinate system origin for all decision regions derived from the received vector. This shift in vector space coordinate systems allowed the integrand of the two-dimensional integral describing the conditional (on the transmitted signal) probability of error to be independent of the transmitted signal. A by-product of Craig's work was a new definite integral form for the Gaussian probability function. In particular, the Gaussian probability function $Q(x)$ ordinarily defined by

$$
Q(x)=\int_{x}^{\infty} \frac{1}{\sqrt{2 \pi}} \exp \left(-\frac{y^{2}}{2}\right) d y
$$

could also now be defined (but only for $x \geq 0$ ) by

$$
Q(x)=\frac{1}{\pi} \int_{0}^{\pi / 2} \exp \left(-\frac{x^{2}}{2 \sin ^{2} \theta}\right) d \theta .
$$

Paper approved by S. G. Wilson, the Editor for Coding Theory and Applications of the IEEE Communications Society. Manuscript received April 7, 1997; revised July 25, 1997. This work was performed at the Jet Propulsion Laboratory, California Institute of Technology, under a contract with the National Aeronautics and Space Administration.

The authors are with the Jet Propulsion Laboratory, California Institute of Technology, Pasadena, CA 91109-8099 USA (e-mail: marvin.k.simon@jpl.nasa.gov; dariush@shannon.jpl.nasa.gov).

Publisher Item Identifier S 0090-6778(98)01927-8.
The form in (2) is not readily obtainable by a change of variables directly in (1). It can, however, be obtained by a straightforward change of variables of a standard known integral involving $Q(x)$, in particular, [10, eq. 3.362(2)]. ${ }^{1}$ In addition to the advantage of having finite integration limits, the form in (2) has the argument of the function $Q(x)$, namely, $x$, contained in the integrand rather than in the integration limits as is the case in (1). The latter has some interesting implications with regard to simplifying the evaluation of performance results related to communication problems wherein the argument of $Q(x)$ is dependent on random system parameters and, thus, requires averaging over the statistics of these parameters. In what follows, we give some examples of such problems with the hope of stimulating further application of the result in (2).

\section{ERror PRobability Performance of UNCODED PSK WITH COSTAS LOOP TRACKING}

It is well known (see [2] for example) that the bit-error probability performance of an uncoded PSK system with an imperfect carrier reference derived from a Costas loop is given $\mathrm{by}^{2}$

$$
P_{b}(E)=\int_{-\pi / 2}^{\pi / 2} P_{b}(E ; \phi) p(\phi) d \phi
$$

where

$$
P_{b}(E ; \phi)=Q\left(\sqrt{\frac{2 E_{b}}{N_{0}}} \cos \phi\right)
$$

is the conditional (on the loop phase error $\phi$ ) bit-error probability and

$$
p(\phi)=\frac{2 \exp \left(\rho_{e q} \cos 2 \phi\right)}{2 \pi I_{0}\left(\rho_{e q}\right)}, \quad-\pi / 2 \leq \phi \leq \pi / 2
$$

is the probability density function (pdf) of the phase error in the form of a Tikhonov distribution. Also, in (4) and (5), $E_{b} / N_{0}$ is the bit-energy-to-noise ratio and

$$
\rho_{e q}=\frac{\rho S_{L}}{4}
$$

\footnotetext{
${ }^{1}$ This standard integral from which (2) can be derived was pointed out to the authors by one of the reviewers.

${ }^{2}$ This result assumes that the $180^{\circ}$ phase ambiguity associated with the Costas loop is perfectly resolved. Methods for accomplishing this are beyond the scope of this discussion.
} 
is the equivalent loop signal-to-noise ratio (SNR) with $\rho=$ $P_{t} T_{b} / N_{0}\left(P_{t}\right.$ denotes the total received power and $T_{b}$ denotes the bit time interval) and

$$
S_{L}=\frac{2 E_{b} / N_{0}}{1+2 E_{b} / N_{0}}
$$

is the so-called squaring loss assuming ideal integrate-anddump arm filters for the Costas loop. Substituting (4) and (5) in (3) results in

$$
P_{b}(E)=\int_{-\pi / 2}^{\pi / 2} Q\left(\sqrt{\frac{2 E_{b}}{N_{0}}} \cos \phi\right) \frac{\exp \left(\rho_{e q} \cos 2 \phi\right)}{\pi I_{0}\left(\rho_{e q}\right)} d \phi
$$

which ordinarily is evaluated by numerical integration using an appropriate subroutine for $Q(x)$ which itself is an integral in accordance with its definition in (1). The evaluation of (8) can be simplified (?) a bit by using the form of $Q(x)$ given in (2). In particular, we obtain the following development:

$$
\begin{aligned}
P_{b}(E)= & \frac{1}{\pi^{2} I_{0}\left(\rho_{e q}\right)} \\
& \cdot \int_{-\pi / 2}^{\pi / 2} \int_{0}^{\pi / 2} \exp \left(-\frac{E_{b}}{N_{0} \sin ^{2} \theta} \cos ^{2} \phi\right) \\
& \cdot \exp \left(\rho_{e q} \cos 2 \phi\right) d \theta d \phi \\
= & \frac{1}{\pi^{2} I_{0}\left(\rho_{e q}\right)} \\
& \cdot \int_{-\pi / 2}^{\pi / 2} \int_{0}^{\pi / 2} \exp \left[-\frac{E_{b}}{2 N_{0} \sin ^{2} \theta}(1+\cos 2 \phi)\right] \\
& \cdot \exp \left(\rho_{e q} \cos 2 \phi\right) d \theta d \phi \\
= & \frac{1}{\pi^{2} I_{0}\left(\rho_{e q}\right)} \int_{0}^{\pi / 2} \exp \left(-\frac{E_{b}}{2 N_{0} \sin ^{2} \theta}\right) \\
& \cdot \int_{-\pi / 2}^{\pi / 2} \exp \left\{\left(-\frac{E_{b}}{2 N_{0} \sin ^{2} \theta}+\rho_{e q}\right) \cos 2 \phi\right\} d \phi d \theta \\
= & \frac{1}{2 \pi^{2} I_{0}\left(\rho_{e q}\right)} \int_{0}^{\pi / 2} \exp \left(-\frac{E_{b}}{2 N_{0} \sin ^{2} \theta}\right) \\
& \cdot \int_{-\pi}^{\pi} \exp \left\{\left(-\frac{E_{b}}{2 N_{0} \sin ^{2} \theta}+\rho_{e q}\right) \cos \Phi\right\} d \Phi d \theta .
\end{aligned}
$$

Finally, recognizing that the integral on $\Phi$ is in the form of a modified Bessel function of the first kind, we get the final desired result

$$
\begin{aligned}
P_{b}(E)= & \frac{1}{\pi} \int_{0}^{\pi / 2} \exp \left(-\frac{E_{b}}{2 N_{0} \sin ^{2} \theta}\right) \\
& \cdot \frac{I_{0}\left(-\frac{E_{b}}{2 N_{0} \sin ^{2} \theta}+\rho_{e q}\right)}{I_{0}\left(\rho_{e q}\right)} d \theta .
\end{aligned}
$$

The form of (10) is interesting in that the $Q(x)$ function needed in the integrand of (8) has been replaced by a modified Bessel function with an argument related to both the equivalent loop $\operatorname{SNR}\left(\rho_{e q}\right)$ and the detection SNR $\left(E_{b} / N_{0}\right)$.
III. Word-ERror Probability Performance of BINARY ANTIPODAL MODULATION WITH INDEPENDENT RAYLEIGH FADING AMPLITUDES-KNOWN CHANNEL-STATE INFORMATION

Consider the transmission of one of two binary digital waveforms (words) over an AWGN channel which is also perturbed by Rayleigh fading. In particular, define the two transmitted signals of duration $N T$ s by

$$
\begin{aligned}
& s_{0}(t)=\sqrt{P} \sum_{n=1}^{N} d_{n} p(t-n T) \\
& s_{1}(t)=-s_{0}(t)
\end{aligned}
$$

where $p(t)$ is a unit amplitude rectangular pulse of duration $T$ s in the interval $0 \leq t \leq T$, and $d_{n}$ takes on values \pm 1 depending on the specific binary data pattern that represents the signals. The additive Gaussian noise $n(t)$ has single-sided power spectral density $N_{0} \mathrm{~W} / \mathrm{Hz}$, and assume that each bit (duration $T \mathrm{~s}$ ) of the signals is independently faded with an identical Rayleigh distribution. As such, the received signal [assuming $s_{0}(t)$ was sent] is expressed as

$$
r(t)=s_{0}^{\prime}(t)+n(t)
$$

where

$$
s_{0}^{\prime}(t)=\sqrt{P} \sum_{n=1}^{N} \rho_{n} d_{n} p(t-n T)
$$

and $\boldsymbol{\rho} \triangleq\left(\rho_{1}, \rho_{2}, \cdots, \rho_{N}\right)$ is an independently identically distributed (i.i.d.) sequence with normalized $\left(E\left\{\rho_{i}^{2}\right\}=1\right)$ Rayleigh pdf

$$
p\left(\rho_{i}\right)=2 \rho_{i} \exp \left(-\rho_{i}^{2}\right), \quad 0 \leq \rho_{i} \leq \infty .
$$

It is straightforward to show that, assuming complete knowledge of the channel fading state, the optimum (maximum $a$ posteriori) receiver implements the decision rule

Choose $s_{0}(t)$ if $\sum_{n=1}^{N} \rho_{n} d_{n} \int_{(n-1) T}^{n T} r(t) d t>0$ otherwise choose $s_{1}(t)$.

Since $X \triangleq \sum_{n=1}^{N} \rho_{n} d_{n} \int_{(n-1) T}^{n T} r(t) d t$ is Gaussian with conditional mean and variance

$$
E\left\{X \mid s_{0}(t)\right\}=\sqrt{P} T \sum_{n=1}^{N} \rho_{n}^{2}, \quad \operatorname{var}\{X\}=\frac{N_{0} T}{2} \sum_{n=1}^{N} \rho_{n}^{2}
$$

then it is easily shown that the conditional average word-error probability based on the above decision rule is given by

$$
P(E \mid \rho)=Q\left(\sqrt{\frac{2 P T}{N_{0}} \sum_{n=1}^{N} \rho_{n}^{2}}\right) .
$$

The unconditional error probability is then obtained by averaging (16) over the $N$ identical pdf's in (14) resulting in the 
$N$-fold integral

$$
\begin{aligned}
P(E)= & \int_{0}^{\infty} \int_{0}^{\infty} \cdots \int_{0}^{\infty} Q\left(\sqrt{\frac{2 P T}{N_{0}} \sum_{n=1}^{N} \rho_{n}^{2}}\right) \\
& \cdot p\left(\rho_{1}\right) p\left(\rho_{2}\right) \cdots p\left(\rho_{N}\right) d \rho_{1} d \rho_{2} \cdots d \rho_{N} .
\end{aligned}
$$

Using (2), the $N$-fold integral with infinite limits of (17) can be reduced to a single integral with finite limits as follows:

$$
\begin{aligned}
P(E)= & \int_{0}^{\infty} \int_{0}^{\infty} \cdots \int_{0}^{\infty} \frac{1}{\pi} \\
& \cdot \int_{0}^{\pi / 2} \exp \left\{-\frac{\left(\sum_{n=1}^{N} \rho_{n}^{2}\right) P T / N_{0}}{\sin ^{2} \theta}\right\} \\
& \cdot\left[\prod_{i=1}^{N} 2 \rho_{i} \exp \left(-\rho_{i}^{2}\right)\right] d \theta d \rho_{1} d \rho_{2} \cdots d \rho_{N} \\
= & \frac{1}{\pi} \int_{0}^{\pi / 2}\left\{\int_{0}^{\infty} 2 \rho \exp \left[-\rho^{2}\left(1+\frac{P T / N_{0}}{\sin ^{2} \theta}\right)\right] d \rho\right\}^{N} d \theta
\end{aligned}
$$

which, when simplified, becomes

$$
P(E)=\frac{1}{\pi} \int_{0}^{\pi / 2}\left(\frac{1}{1+\frac{P T / N_{0}}{\sin ^{2} \theta}}\right)^{N} d \theta .
$$

For the Rayleigh-fading case as considered here, evaluation of (17) could be simplified by recognizing that the $N$-fold average can be looked upon as a single average over the chisquared random variable $\sum_{n=1}^{N} \rho_{n}^{2}$. Thus, in this instance, (19) may not be that much simpler than (17) although the former is still an integral with finite limits whereas the latter would become an integral over a semi-infinite interval. However, in the more general i.i.d. fading case where no simple expression exists for the pdf of $\sum_{n=1}^{N} \rho_{n}^{2}$, the technique of applying (2) to the Gaussian integral in (17) will always, regardless of the fading pdf, reduce to a single integral of some function of $\theta$ raised to the $N$ th power analogous to (19).

\section{Bit-ERror Probability of CODED MPSK Signaling Over a MEMORYLESS FADING CHANNEL- KNOWN CHANNEL-STATE INFORMATION}

The previous example can be considerably generalized to yield similar benefits. In particular, consider the transmission of coded (MPSK) signals over an AWGN channel which is also perturbed by fading. ${ }^{3}$ If the fading is independent from transmission to transmission then the resulting channel is memoryless. An example of the performance evaluation for such an example was considered in [3] where the error correction coding was specifically trellis coding. The reader is referred to that paper for the details of the analysis. In

\footnotetext{
${ }^{3}$ Note that we are not restricting the fading statistics to be Rayleigh distributed. In fact, later we shall show that simple results are obtainable for Rician as well as Rayleigh fading.
}

short, the bit-error performance was derived in the form of a union-Chernoff bound where the Chernoff bound portion applied to the pairwise error probability and the union bound portion converted the pairwise error probability to average biterror probability using the transfer function bound method. We shall show here that using the alternate form of $Q(x)$ given in (2) enables one to eliminate the need for Chernoff bounding the pairwise error probability. ${ }^{4}$ Hence, the resulting form for the average bit-error probability is strictly a union (transfer function) bound.

Following [3], we denote a coded MPSK symbol sequence of length $N$ by ${ }^{5}$

$$
\mathbf{x}=\left(x_{1}, x_{2}, \cdots, x_{N}\right)
$$

where the $k$ th element of $\mathbf{x}$, namely, $x_{k}$, represents the transmitted MPSK symbol at time $k$ and is a nonlinear function of the state of the encoder $s_{k}$ and the $b$ information bits $\mathbf{u}_{k}$ at its input, i.e., $x_{k}=f\left(s_{k}, \mathbf{u}_{k}\right)$. The transition from state to state is defined by a similar nonlinear relation, namely, $s_{k+1}=g\left(s_{k}, \mathbf{u}_{k}\right)$. Corresponding to the transmission of $\mathbf{x}$, the channel outputs the sequence

$$
\mathbf{y}=\left(y_{1}, y_{2}, \cdots, y_{N}\right)
$$

where the $k$ th element of $\mathbf{y}$, namely, $y_{k}$, representing the channel output at time $k$, is given by

$$
y_{k}=\rho_{k} x_{k}+n_{k} \text {. }
$$

As before, $\rho_{k}$ is the normalized fading amplitude for the $k$ th transmission and $n_{k}$ is a zero-mean complex Gaussian random variable with variance $\sigma^{2}$ per dimension.

For the case of known channel-state information, it was shown in [3] that using the maximum-likelihood decision metric for i.i.d. fading per transmission, the conditional (on the channel-state information) pairwise error probability, namely, the probability of deciding $\hat{\mathbf{x}}$ when indeed $\mathrm{x}$ was transmitted, is given by

$$
P(\mathbf{x} \rightarrow \hat{\mathbf{x}} \mid \boldsymbol{\rho})=Q\left(\frac{1}{2 \sigma} \sqrt{\sum_{n \in \eta} \rho_{n}^{2}\left|\hat{x}_{n}-x_{n}\right|^{2}}\right)
$$

where $\eta$ is the set of all $n$ for which $\hat{x}_{n} \neq x_{n}$.

Using the form of $Q(x)$ given in (2), we can express (23) as

$$
\begin{aligned}
P(\mathbf{x} \rightarrow \hat{\mathbf{x}} \mid \rho) & =\frac{1}{\pi} \int_{0}^{\pi / 2} \exp \left\{-\frac{\sum_{n \in \eta} \rho_{n}^{2}\left|\hat{x}_{n}-x_{n}\right|^{2}}{8 \sigma^{2} \sin ^{2} \theta}\right\} d \theta \\
& =\frac{1}{\pi} \int_{0}^{\pi / 2}[D(\theta)]^{d^{2}(\mathbf{x}, \hat{\mathbf{x}})} d \theta
\end{aligned}
$$

where

$$
D(\theta) \triangleq \exp \left\{-\frac{1}{8 \sigma^{2} \sin ^{2} \theta}\right\}
$$

\footnotetext{
${ }^{4}$ A similar method using a different representation of $Q(x)$ but with infinite range is discussed in [7].

${ }^{5}$ We assume that the MPSK symbols are normalized such that $\left|x_{n}\right|=1$, i.e., the signals lie on the perimeter of the unit circle.
} 
and

$$
d^{2}(\mathbf{x}, \hat{\mathbf{x}}) \triangleq \sum_{n \in \eta} \rho_{n}^{2}\left|\hat{x}_{n}-x_{n}\right|^{2}
$$

represents the square of the weighted Euclidean distance between the two symbol sequences. Alternately

$$
P(\mathbf{x} \rightarrow \hat{\mathbf{x}} \mid \boldsymbol{\rho})=\frac{1}{\pi} \int_{0}^{\pi / 2} \prod_{n \in \eta}[D(\theta)]^{\rho_{n}^{2}\left|\hat{x}_{n}-x_{n}\right|^{2}} d \theta .
$$

The exact form of (24) is to be compared with the Chernoff bound given by [3, eq. (20)], namely

$$
P(\mathbf{x} \rightarrow \hat{\mathbf{x}} \mid \rho) \leq D^{d^{2}(\mathbf{x}, \hat{\mathbf{x}})}
$$

where

$$
D \triangleq \exp \left\{-\frac{1}{8 \sigma^{2}}\right\}
$$
by

Finally, the unconditional pairwise error probability is given

$$
\begin{aligned}
P(\mathbf{x} \rightarrow \hat{\mathbf{x}}) & =\overline{\frac{1}{\pi} \int_{0}^{\pi / 2}[D(\theta)]^{d^{2}(\mathbf{x}, \hat{\mathbf{x}})} d \theta} \boldsymbol{\rho} \\
& =\frac{1}{\pi} \int_{0}^{\pi / 2} \overline{[D(\theta)]^{d^{2}(\mathbf{x}, \hat{\mathbf{x}})}} \boldsymbol{\rho} d \theta
\end{aligned}
$$

where the overbar denotes statistical averaging over the vector random variable $\boldsymbol{\rho}$. Furthermore, since the $\rho_{n}$ 's are i.i.d., then the average on $\boldsymbol{\rho}$ can be partitioned with the result that

$$
P(\mathrm{x} \rightarrow \hat{\mathbf{x}})=\frac{1}{\pi} \int_{0}^{\pi / 2} \prod_{n \in \eta} \overline{[D(\theta)]^{\rho_{n}^{2}\left|\hat{x}_{n}-x_{n}\right|^{2}}} \rho_{n} d \theta .
$$

Using the pair-state method discussed in [3], the exact pairwise error probability of (30) or (31) can be converted to a union bound on the average bit-error probability. In particular, a pair-state transition diagram [4] is constructed in terms of the pair state

$$
S_{k} \triangleq\left(s_{k}, \hat{s}_{k}\right), \quad U_{k} \triangleq\left(u_{k}, \hat{u}_{k}\right)
$$

where $\hat{s}_{k}, \hat{u}_{k}$ are, respectively, the estimates of the state of the decoder and the information symbol. Using the definition of $x_{n}$ and rewriting (26) as

$$
\begin{aligned}
d^{2}(\mathbf{x}, \hat{\mathbf{x}}) & =\sum_{n \in \eta} \rho_{n}^{2}\left|f\left(s_{n}, \mathbf{u}_{n}\right)-f\left(\hat{s}_{n}, \hat{\mathbf{u}}_{n}\right)\right|^{2} \\
& \triangleq \sum_{n \in \eta} \delta^{2}\left(S_{n}, U_{n}\right)
\end{aligned}
$$

then, by analogy with results in [3] and [4], the average bit-error probability is upper bounded by

$$
P_{b}(E) \leq\left.\frac{1}{\pi} \int_{0}^{\pi / 2} \frac{1}{b} \frac{d}{d I} T[D(\theta), I]\right|_{I=1} d \theta
$$

where $T[D(\theta), I]$ is the transfer function of the pairstate transition diagram whose branch labels contain the

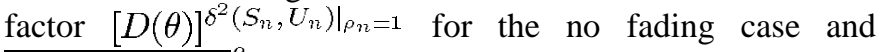

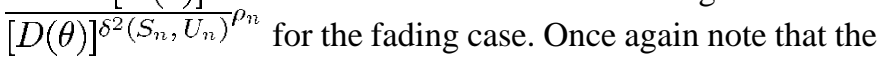
Chernoff bound on the pairwise error probability previously required in order to apply the transfer function bound has been eliminated and instead replaced by a single integration on the variable $\theta$ after evaluating the transfer function. In this sense, the form of $Q(x)$ in (2) allows manipulations akin to those afforded by the Chernoff bound but without the necessity of invoking a bound. Actually, this observation can be made directly from (2) by noting that the integrand has its maximum value when $\theta=\pi / 2$. Thus, replacing the integrand by its maximum value we get the well-known upper bound on $Q(x)$, namely, $Q(x) \leq \frac{1}{2} \exp \left(-x^{2} / 2\right)$, which is in the form of a Chernoff bound.

The average on $\rho_{n}$ required in (31) is easily evaluated for Rayleigh- and Rician-fading channels. In particular, using the results in [3] and letting $1 / 2 \sigma^{2}=E_{s} / N_{0}$, we have for the Rayleigh pdf of (14)

$$
\overline{[D(\theta)]^{2}\left|\hat{x}_{n}-x_{n}\right|^{2}} \rho_{n n}=\frac{1}{1+\frac{\beta_{n} \gamma}{\sin ^{2} \theta}}
$$

where as in [3]

$$
\beta_{n} \triangleq\left|\hat{x}_{n}-x_{n}\right|^{2}, \quad \gamma \triangleq \bar{E}_{s} / 4 N_{0} .
$$

Similarly, for the Rician channel characterized by the pdf

$$
\begin{aligned}
p\left(\rho_{i}\right)= & 2 \rho_{i}(1+K) \exp \left[-K-\rho^{2}(1+K)\right] \\
& \cdot I_{0}\left[2 \rho_{i} \sqrt{K(1+K)}\right], \quad 0 \leq \rho_{i} \leq \infty
\end{aligned}
$$

we have

$$
\begin{aligned}
& \overline{[D(\theta)]^{\rho_{n}^{2}\left|\hat{x}_{n}-x_{n}\right|^{2}}} \rho_{n} \\
& =\left(\frac{1+K}{1+K+\frac{\beta_{n} \gamma}{\sin ^{2} \theta}}\right) D(\theta)^{\beta_{n} K /\left(1+K+\beta_{n} \gamma / \sin ^{2} \theta\right)}
\end{aligned}
$$

which clearly simplifies to (36) for $K=0$. Note that for binary PSK (BPSK) (i.e., $M=2$ ) we have $\beta_{n}=4$ independent of $n$ and, thus, for the Rayleigh case, for example, (31) simplifies to ${ }^{6}$

$$
P(\mathbf{x} \rightarrow \hat{\mathbf{x}})=\frac{1}{\pi} \int_{0}^{\pi / 2}\left[\frac{1}{1+\frac{\bar{E}_{s} / N_{0}}{\sin ^{2} \theta}}\right]^{d} d \theta
$$

where $d$ is the cardinality of $\eta$, i.e., the Hamming distance between $\mathbf{x}$ and $\hat{\mathbf{x}}$. If the two code words are equal and opposite, then $d=N$ and (39) agrees with (19).

Application of (34) for specific trellis codes can be easily carried out using the examples given in [3].

\section{Conditional Symbol ERror Probability of MPSK IN THE PRESENCE OF CARRIER SYNCHRONIZATION ERROR}

Consider a coherent MPSK system with a carrier tracking loop that produces a phase error $\phi$. The conditional (on $\phi$ )

\footnotetext{
${ }^{6} \mathrm{~A}$ similar result was obtained in [8].
} 
symbol-error probability of such a system has been previously computed in [2, Appendix B] and is given by

$$
\begin{aligned}
P(E ; \phi)= & \frac{M-1}{M}-\frac{1}{4} \operatorname{erf}\left[\sqrt{\frac{E_{s}}{N_{0}}} \sin \left(\frac{\pi}{M}+\phi\right)\right] \\
& -\frac{1}{4} \operatorname{erf}\left[\sqrt{\frac{E_{s}}{N_{0}}} \sin \left(\frac{\pi}{M}-\phi\right)\right] \\
& -\frac{1}{2 \sqrt{\pi}} \int_{0}^{\sqrt{E_{s} / N_{0}} \sin (\pi / M+\phi)} \exp \left(-y^{2}\right) \\
& \cdot \operatorname{erf}\left[y \cot \left(\frac{\pi}{M}+\phi\right)\right] d y \\
& -\frac{1}{2 \sqrt{\pi}} \int_{0}^{\sqrt{E_{s} / N_{0}} \sin (\pi / M-\phi)} \exp \left(-y^{2}\right) \\
& \cdot \operatorname{erf}\left[y \cot \left(\frac{\pi}{M}-\phi\right)\right] d y .
\end{aligned}
$$

We now show that this expression can be simplified using the alternate form of $Q(x)$ given in (2).

The vector representation of an MPSK system consists of $M$ points uniformly distributed on a circle of radius $\sqrt{E_{s}}$. For this system, the geometry for the correct decision region associated with the transmitted signal point $\mathbf{s}_{0}=-\sqrt{E_{s}}$ is illustrated in Fig. 1 where, as suggested in [1], the origin of coordinates has been shifted to the signal point. Also note that for convenience we have rotated the coordinate system by $\phi$ radians. Following the approach in [1] (also see [5, Ch. 3, Section 3.2.8]), we can write the probability of an error given that signal $\mathbf{s}_{0}$ is transmitted as

$$
\begin{aligned}
P\left(E ; \phi \mid \mathbf{s}_{0}\right)= & \int_{0}^{\pi-(\pi / M+\phi)} d \theta \int_{R_{1}}^{\infty} p_{R, \Theta}(r, \theta) d r \\
& +\int_{-\pi+(\pi / M-\phi)}^{0} d \theta \int_{R_{2}}^{\infty} p_{R, \Theta}(r, \theta) d r
\end{aligned}
$$

where $R$ is the distance from the signal point to the boundary point $E$ (in general, a function of $\theta$ ) and $p_{R, \Theta}(r, \theta)$ is the bivariate Gaussian pdf that represents the noise vector in polar coordinates, that is

$$
p_{R, \Theta}(r, \theta)=\frac{r}{\pi N_{0}} \exp \left(-\frac{r^{2}}{N_{0}}\right), \quad 0 \leq r \leq \infty
$$

which is clearly independent of $\theta$. Substituting (42) into (41) and performing the integration on $r$ gives the simplification

$$
\begin{aligned}
P\left(E ; \phi \mid \mathbf{s}_{0}\right)= & \int_{0}^{(M-1) \pi / M-\phi} \frac{1}{2 \pi} \exp \left(-\frac{R_{1}^{2}}{N_{0}}\right) d \theta \\
& +\int_{-[(M-1) \pi / M]-\phi}^{0} \frac{1}{2 \pi} \exp \left(-\frac{R_{2}^{2}}{N_{0}}\right) d \theta
\end{aligned}
$$

Applying the law of sines to triangles $O O^{\prime} E$ and $O O^{\prime} E^{\prime}$, we get

$$
\begin{aligned}
& \frac{R_{1}}{\sqrt{E_{s}}}=\frac{\sin \left(\frac{\pi}{M}+\phi\right)}{\sin \left(\frac{\pi}{M}+\phi+\theta\right)} \\
& \frac{R_{2}}{\sqrt{E_{s}}}=\frac{\sin \left(\frac{\pi}{M}-\phi\right)}{\sin \left(\frac{\pi}{M}-\phi-\theta\right)} .
\end{aligned}
$$

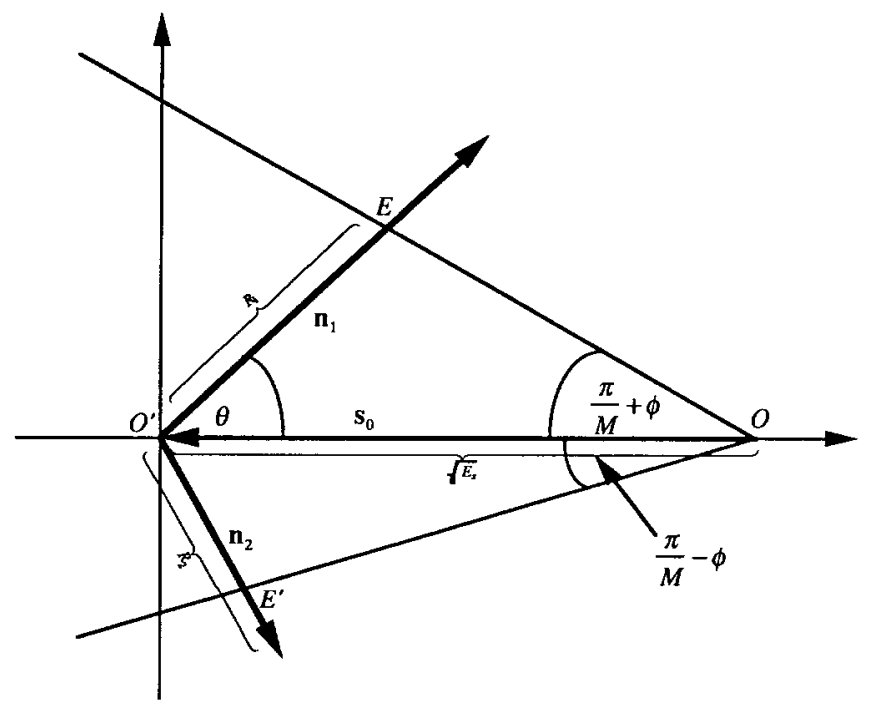

Fig. 1. Geometry for correct decision region for $\mathbf{s}_{0}$.

Combining (44) with (43) and simplifying using appropriate changes of variables gives the final desired result for the conditional symbol-error probability, namely

$$
\begin{aligned}
& P(E ; \phi) \\
& =\frac{1}{2 \pi} \int_{0}^{(M-1) \pi / M-\phi} \exp \left[-\frac{E_{s}}{N_{0}} \frac{\sin ^{2}\left(\frac{\pi}{M}+\phi\right)}{\sin ^{2} \Phi}\right] d \Phi \\
& \quad+\frac{1}{2 \pi} \int_{0}^{(M-1) \pi / M+\phi} \exp \left[-\frac{E_{s}}{N_{0}} \frac{\sin ^{2}\left(\frac{\pi}{M}-\phi\right)}{\sin ^{2} \Phi}\right] d \Phi
\end{aligned}
$$

where we have also taken note of the fact that from the symmetry of the signal constellation, $P\left(E ; \phi \mid \mathbf{s}_{i}\right)$ is independent of $i$. Note that no error functions are needed to evaluate (45). For $M=2$ (BPSK), (45) simplifies to

$$
\begin{aligned}
\left.P(E ; \phi)\right|_{M=2}= & \frac{1}{\pi} \int_{0}^{\pi / 2} \exp \left(-\frac{E_{s}}{N_{0}} \frac{\cos ^{2} \phi}{\sin ^{2} \Phi}\right) d \Phi \\
& -\frac{1}{2 \pi} \int_{\pi / 2-\phi}^{\pi / 2} \exp \left(-\frac{E_{s}}{N_{0}} \frac{\cos ^{2} \phi}{\sin ^{2} \Phi}\right) d \Phi \\
& +\frac{1}{2 \pi} \int_{\pi / 2}^{\pi / 2+\phi} \exp \left(-\frac{E_{s}}{N_{0}} \frac{\cos ^{2} \phi}{\sin ^{2} \Phi}\right) d \Phi .
\end{aligned}
$$

Making the changes of variables $\alpha=-(\Phi-\pi / 2), \beta=$ $\Phi-\pi / 2$ in the second and third integrals of (46), respectively, we see that these terms cancel and thus

$$
\begin{aligned}
\left.P(E ; \phi)\right|_{M=2} & =\frac{1}{\pi} \int_{0}^{\pi / 2} \exp \left(-\frac{E_{s}}{N_{0}} \frac{\cos ^{2} \phi}{\sin ^{2} \Phi}\right) d \Phi \\
& =Q\left(\sqrt{\frac{2 E_{s}}{N_{0}}} \cos \phi\right)
\end{aligned}
$$

which is the well-known result used in Example 1. 


\section{EVALUATION OF A WELL-KNOWN INTEGRAL}

Consider the integral

$$
I=\int_{C_{1}}^{C_{2}} \exp \left(-a^{2} z^{2} \pm b z\right) \operatorname{erfc} z d z
$$

where $\operatorname{erfc} z$ is the complementary error function with argument $z$ and $C_{1}, C_{2}$ are constants which, in many cases of interest, are either zero or infinite. This integral occurs, for example, in problems where average error probability performance is to be computed in the presence of Gaussian interference other than that produced by the additive noise. For the special case of $C_{1}=-\infty, C_{2}=\infty$ this integral can be evaluated in closed form as [6, Appendix 1, Eq. (A.1.10a)]

$$
\begin{aligned}
\int_{-\infty}^{\infty} \exp \left(-a^{2} z^{2}\right. & \pm b z) \operatorname{erfc} z d z \\
= & \frac{\sqrt{\pi}}{a} \exp \left(\frac{b^{2}}{4 a^{2}}\right) \operatorname{erfc}\left[\frac{ \pm b}{2 a \sqrt{a^{2}+1}}\right] .
\end{aligned}
$$

For any other pair of values $C_{1}, C_{2}$ a closed form for this integral has not been found. Although we too cannot find a closed form for the general case of (48), we are, however, able to convert the integral into one of fixed finite limits which therefore simplifies the case where one of the two constants $C_{1}, C_{2}$ is infinite, e.g., $C_{1}=0, C_{2}=\infty$.

Rewriting (48) in terms of the Gaussian probability integral and using (2), we get

$$
\begin{aligned}
I & =2 \int_{C_{1}}^{C_{2}} Q(\sqrt{2 z}) \exp \left(-a^{2} z^{2} \pm b z\right) d z \\
& =\frac{2}{\pi} \int_{0}^{\pi / 2} \int_{C_{1}}^{C_{2}} \exp \left[-\left(a^{2}+\frac{1}{\sin ^{2} \theta}\right) z^{2} \pm b z\right] d z d \theta .
\end{aligned}
$$

Completing the square of the argument of the exponential gives

$$
\begin{aligned}
I= & \frac{2}{\pi} \int_{0}^{\pi / 2} \exp \left[-\frac{b^{2}}{2 A^{2}(\theta)}\right] \\
& \cdot \int_{C_{1}}^{C_{2}} \exp \left\{-\left[A(\theta) z \pm \frac{b}{2 A(\theta)}\right]\right\} d z d \theta
\end{aligned}
$$

where

$$
A(\theta)=\sqrt{a^{2}+\frac{1}{\sin ^{2} \theta}} .
$$

Performing the integration on $z$ gives the final desired result

$$
\begin{aligned}
I= & \frac{1}{\pi} \int_{0}^{\pi / 2} \frac{\sqrt{\pi}}{A(\theta)} \exp \left[-\frac{b^{2}}{2 A^{2}(\theta)}\right] \\
& \cdot\left\{\operatorname{erfc}\left[C_{1} A(\theta) \pm \frac{b}{2 A(\theta)}\right]-\operatorname{erfc}\left[C_{2} A(\theta) \pm \frac{b}{2 A(\theta)}\right]\right\} d \theta .
\end{aligned}
$$

As an example, consider the special case where $C_{1}=0, C_{2}=$ $\infty$. Then, since $\operatorname{erfc}(\infty)=0$, we have

$$
\begin{aligned}
I & =\int_{0}^{\infty} \exp \left(-a^{2} z^{2} \pm b z\right) \operatorname{erfc} z d z \\
& =\frac{1}{\pi} \int_{0}^{\pi / 2} \frac{\sqrt{\pi}}{A(\theta)} \exp \left[-\frac{b^{2}}{2 A^{2}(\theta)}\right] \operatorname{erfc}\left[ \pm \frac{b}{2 A(\theta)}\right] d \theta .
\end{aligned}
$$

It is to be emphasized that (54) is not readily obtainable by any straightforward change of variables in (48).

\section{Average ERror Probability for the Binary AWGN INTERSYMBOL INTERFERENCE (ISI) CHANNEL}

It is well known [9] that maximum-likelihood demodulation of binary equiprobable data transmitted over an AWGN channel with ISI of finite memory $L$ can be based on a $2^{L-1}$ state trellis where the states are determined by the preceding $L-1$ data symbols. The algorithm for selecting the most probable sequence is the well known maximum-likelihood decoding (Viterbi) algorithm. The evaluation of the performance of such a demodulator has in the past been expressed in terms of a union-Chernoff (upper) bound on the average error probability [9]. As in Example 4, we shall once again show how the Chernoff portion of the bound can be eliminated by instead using an exact expression based on (2) for the pairwise error probability which, in this example, also corresponds to the probability of choosing a particular incorrect path in the trellis rather than the correct one.

Consider a binary data source characterized by the impulse sequence

$$
d(t)=\sum_{k=-\infty}^{\infty} d_{k} \delta(t-k T)
$$

where, as before, $\left\{d_{k}\right\}$ is a binary i.i.d. sequence taking on values \pm 1 . Before transmission over the AWGN channel, the data source is passed through a transmit filter with impulse response $h(t)$. Thus, the transmitted message (signal) is described by

$$
x(t)=\sum_{k=-\infty}^{\infty} d_{k} h(t-k T)
$$

and the corresponding received signal is $y(t)=x(t)+n(t)$. Assuming a maximum-likelihood decision rule, then it has been shown [9] that the pairwise probability $P(\mathbf{x} \rightarrow \hat{\mathbf{x}})$, namely, the probability of choosing the incorrect transmitted sequence [uniform samples of $x(t)$ spaced by $T \mathrm{~s}$ ] $\hat{\mathrm{x}}$ when in fact $\mathbf{x}$ was transmitted, is given by

$$
P(\mathbf{x} \rightarrow \hat{\mathbf{x}})=Q\left[\sqrt{\frac{2}{N_{0}}} \sum_{k=-N}^{N-1}\left(\varepsilon_{k}^{2} h_{0}+2 \sum_{i=1}^{L-1} \varepsilon_{k} \varepsilon_{k-i} h_{i}\right)\right]
$$

where $\left\{h_{i}\right\}$ are the ISI coefficients defined by

$$
h_{k-j} \triangleq \int_{-\infty}^{\infty} h(t-k T) h(t-j T)=h_{j-k}
$$

and $\left\{\varepsilon_{k}\right\}$ are the error sequences defined by

$$
\varepsilon_{k} \triangleq \frac{1}{2}\left(d_{k}-\hat{d}_{k}\right)= \begin{cases}1, & d_{k}=1, \hat{d}_{k}=-1 \\ 0, & d_{k}=\hat{d}_{k} \\ -1, & d_{k}=-1, \hat{d}_{k}=1 .\end{cases}
$$

Rather than use a Chernoff bound on (57) (as was done in [9]), one can again use the form of $Q(x)$ in (2) to write the pairwise error probability as given in (60), shown at the bottom of the next page, which, as before, becomes the Chernoff 


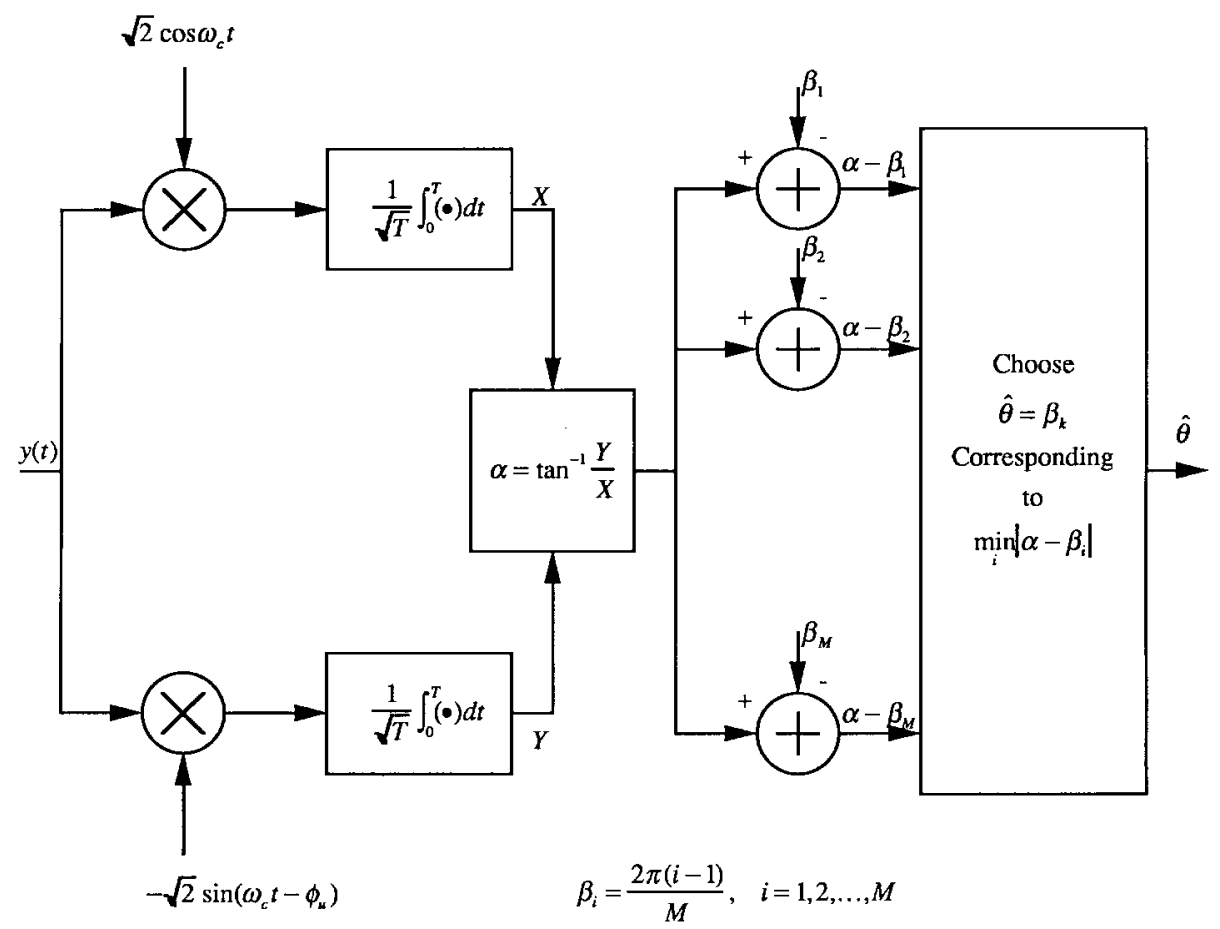

Fig. 2. Optimum MPSK receiver.

bound by letting the integrand take on its maximum value corresponding to $\theta=\pi / 2$. Thus, following steps identical to those in [9, Ch. 4], we arrive at a union bound on the average bit-error probability analogous to the union-Chernoff bound given by [9, eq. (4.9.23)], namely

$$
\begin{aligned}
P_{b}(E) \leq & \sum_{\varepsilon} w(\varepsilon) \frac{1}{\pi} \int_{0}^{\pi / 2} \prod_{k=-N}^{N-1} \frac{1}{2^{w(\varepsilon)}} \\
& \cdot \exp \left\{-\frac{1}{N_{0} \sin ^{2} \theta}\left(\varepsilon_{k}^{2} h_{0}+2 \sum_{i=1}^{L-1} \varepsilon_{k} \varepsilon_{k-i} h_{i}\right)\right\} d \theta
\end{aligned}
$$

where $w(\boldsymbol{\varepsilon})$ is the weight (number of nonzero components) of the sequence $\varepsilon$ with components as in (59). The first sum in (61) represents averaging over all possible error sequences. The evaluation of (61) can be carried out by the transfer function bound approach analogous to that used in Section IV.

\section{SyMbol ERror Probability OF MPSK WITH $I-Q$ Unbalance (Detection IN the PRESENCE of CORRELATED QUadRATURE NOISE COMPONENTS)}

Consider a coherent MPSK system with $I-Q$ carrier demodulation reference signals that are not in perfect phase quadrature. In particular, consider the optimum MPSK receiver illustrated in Fig. 2 where the $I$ and $Q$ demodulation reference signals are given by

$$
r_{c}(t)=\sqrt{2} \cos \omega_{c} t \quad r_{s}(t)=-\sqrt{2} \sin \left(\omega_{c} t-\phi_{u}\right)
$$

where $\phi_{u}$ represents the degree of unbalance, i.e., the deviation from perfect phase quadrature. In response to a transmitted MPSK signal

$$
s(t)=\sqrt{2 P} \cos \left(\omega_{c} t+\theta_{m}\right)
$$

where $\theta_{m}$ takes on values $\beta_{i}=2 \pi(i-1) / M, i=1,2, \cdots, M$ with equal probability $1 / M$, the $I$ and $Q$ integrate-and-dump output signals become

$$
\begin{aligned}
X= & \overbrace{\sqrt{E_{s}} \cos \theta_{m}}^{\bar{X}}+\overbrace{\frac{1}{\sqrt{T}} \int_{0}^{T} n(t) \sqrt{2} \cos \omega_{c} t d t}^{N_{X}} \\
Y= & \overbrace{\sqrt{E_{s}} \sin \left(\theta_{m}+\phi_{u}\right)}^{\bar{Y}} \overbrace{\overbrace{\frac{1}{\sqrt{T}} \int_{0}^{T} n(t)\left[-\sqrt{2} \sin \left(\omega_{c} t-\phi_{u}\right)\right] d t}^{N_{Y}}}
\end{aligned}
$$

$$
\begin{aligned}
P(\mathbf{x} \rightarrow \hat{\mathbf{x}}) & =\frac{1}{\pi} \int_{0}^{\pi / 2} \exp \left[-\frac{1}{N_{0} \sin ^{2} \theta} \sum_{k=-N}^{N-1}\left(\varepsilon_{k}^{2} h_{0}+2 \sum_{i=1}^{L-1} \varepsilon_{k} \varepsilon_{k-i} h_{i}\right)\right] d \theta \\
& =\frac{1}{\pi} \int_{0}^{\pi / 2} \prod_{k=-N}^{N-1} \exp \left\{-\frac{1}{N_{0} \sin ^{2} \theta}\left(\varepsilon_{k}^{2} h_{0}+2 \sum_{i=1}^{L-1} \varepsilon_{k} \varepsilon_{k-i} h_{i}\right)\right\} d \theta
\end{aligned}
$$


where $E_{s}=P T$ is the energy per MPSK symbol. The $I$ and $Q$ noises $N_{X}, N_{Y}$ in (64) are zero-mean correlated Gaussian random variables, each with variance $\sigma^{2}=N_{0} / 2$ and correlation

$$
E\left\{N_{X} N_{Y}\right\}=\sigma^{2} \sin \phi_{u} \triangleq \rho \sigma^{2} .
$$

Analogous to the MPSK decision problem with perfect $I-Q$ demodulation, the calculation of the symbol-error probability in the presence of $I-Q$ unbalance can be determined from the geometry of Fig. 3. Assuming, as before, that the transmitted signal is $\mathbf{s}_{0}=-\sqrt{E_{s}}$ (i.e., $\theta_{m}=-\pi$ ), then the evaluation can be carried out either assuming the point $O$ as the center of coordinates (the classical approach) or by the simpler approach of using the point $O^{\prime}$ (the location of the tip of the signal vector) as the center of coordinates. In what follows, we shall assume the latter. Since for the assumed transmitted signal we have from (64) and (65) that $\bar{X}=-\sqrt{E_{s}}, \bar{Y}=-\rho \sqrt{E_{s}}$, then defining the quantities

$$
\begin{aligned}
& \bar{S} \triangleq \sqrt{\bar{X}^{2}+\bar{Y}^{2}}=\sqrt{E_{s}\left(1+\rho^{2}\right)} \\
& \phi_{s}=\tan ^{-1} \frac{\bar{Y}}{\bar{X}}=\tan ^{-1} \rho
\end{aligned}
$$

we have, by analogy with (41), that the probability of error is given by

$$
\begin{aligned}
P\left(E ; \phi_{s} \mid \mathbf{s}_{0}\right)= & \int_{\phi_{s}}^{(M-1) \pi / M} d \theta \int_{R_{1}}^{\infty} p_{R, \Theta}(r, \theta) d r \\
& +\int_{-[(M-1) \pi / M]}^{\phi_{s}} d \theta \int_{R_{2}}^{\infty} p_{R, \Theta}(r, \theta) d r
\end{aligned}
$$

where, as before, $R$ is the distance from the signal point to the boundary point $E$ (in general, a function of $\theta$ ) and $p_{R, \Theta}(r, \theta)$ is the bivariate Gaussian pdf that represents the correlated noise vector in polar coordinates, that is

$$
\begin{array}{r}
p_{R, \Theta}(r, \theta)=\frac{r}{\pi N_{0} \sqrt{1-\rho^{2}}} \exp \left\{-\frac{r^{2}}{N_{0}}\left(\frac{1-\rho \sin 2 \theta}{1-\rho^{2}}\right)\right\}, \\
0 \leq r \leq \infty,-\pi \leq \theta \leq \pi .
\end{array}
$$

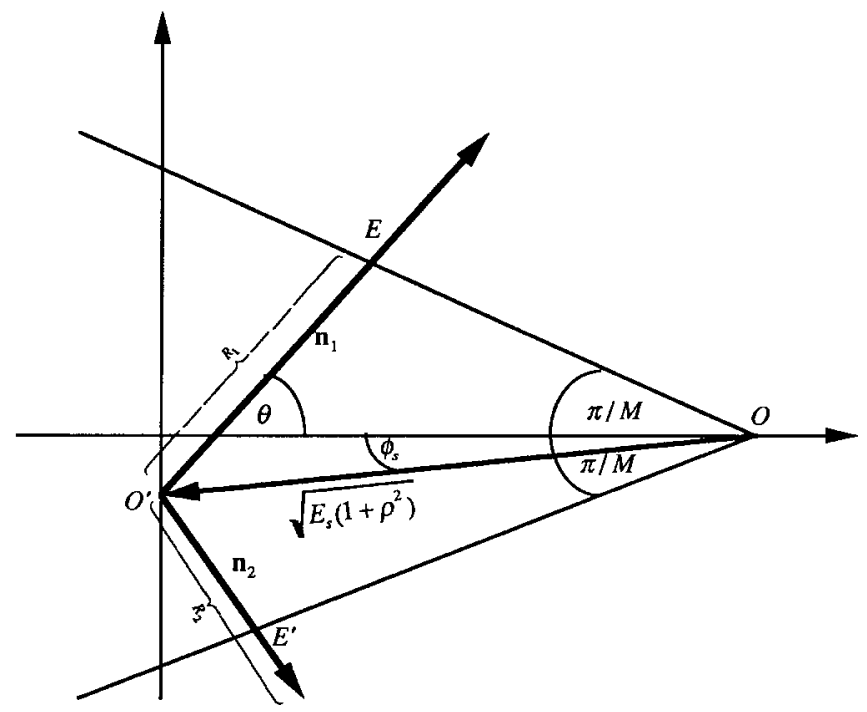

Fig. 3. Geometry for correct decision region for $\mathbf{s}_{0}$.

Applying the law of sines to triangles $O O^{\prime} E$ and $O O^{\prime} E^{\prime}$ in Fig. 3, we get

$$
\begin{aligned}
& \frac{R_{1}}{\sqrt{E_{s}\left(1+\rho^{2}\right)}}=\frac{\sin \left(\frac{\pi}{M}+\phi_{s}\right)}{\sin \left(\frac{\pi}{M}+\theta\right)} \\
& \frac{R_{2}}{\sqrt{E_{s}\left(1+\rho^{2}\right)}}=\frac{\sin \left(\frac{\pi}{M}-\phi_{s}\right)}{\sin \left(\frac{\pi}{M}-\theta\right)} .
\end{aligned}
$$

Combining (69) with (68) and simplifying using appropriate changes of variables gives the final desired result for the symbol-error probability in the presence of $I-Q$ unbalance, namely, as in (70), shown at the bottom of the page, where we have again made use of the symmetry of the signal constellation to note that $P\left(E ; \phi_{s} \mid \mathbf{s}_{i}\right)$ is independent of $i$. The importance of the form in (70) is that the dependence on $E_{s} / N_{0}$ is still in the argument of the exponential of the integrand and, thus, it is straightforward to extend this result to, for example, coded modulation without the necessity of invoking Chernoff bounds.

$$
\begin{aligned}
P\left(E ; \phi_{u}\right)= & \frac{1}{2 \pi} \int_{0}^{(M-1) \pi / M-\phi_{s}} \frac{\sqrt{1-\rho^{2}}}{1+\rho \sin \left[2\left(\Phi+\frac{\pi}{M}\right)\right]} \\
& \cdot \exp \left(-\frac{E_{s}}{N_{0}}\left(\frac{1+\rho^{2}}{1-\rho^{2}}\right) \frac{\left\{1+\rho \sin \left[2\left(\Phi+\frac{\pi}{M}\right)\right]\right\} \sin ^{2}\left(\frac{\pi}{M}+\phi_{s}\right)}{\sin ^{2} \Phi}\right) d \Phi \\
& +\frac{1}{2 \pi} \int_{0}^{(M-1) \pi / M+\phi_{s}} \frac{\sqrt{1-\rho^{2}}}{1-\rho \sin \left[2\left(\Phi+\frac{\pi}{M}\right)\right]} \\
& \cdot \exp \left(-\frac{E_{s}}{N_{0}}\left(\frac{1+\rho^{2}}{1-\rho^{2}}\right) \frac{\left\{1-\rho \sin \left[2\left(\Phi+\frac{\pi}{M}\right)\right]\right\} \sin ^{2}\left(\frac{\pi}{M}-\phi_{s}\right)}{\sin ^{2} \Phi}\right) d \Phi \\
\rho \triangleq & \sin \phi_{u}, \quad \phi_{s}=\tan ^{-1} \rho=\tan ^{-1}\left(\sin \phi_{u}\right)
\end{aligned}
$$




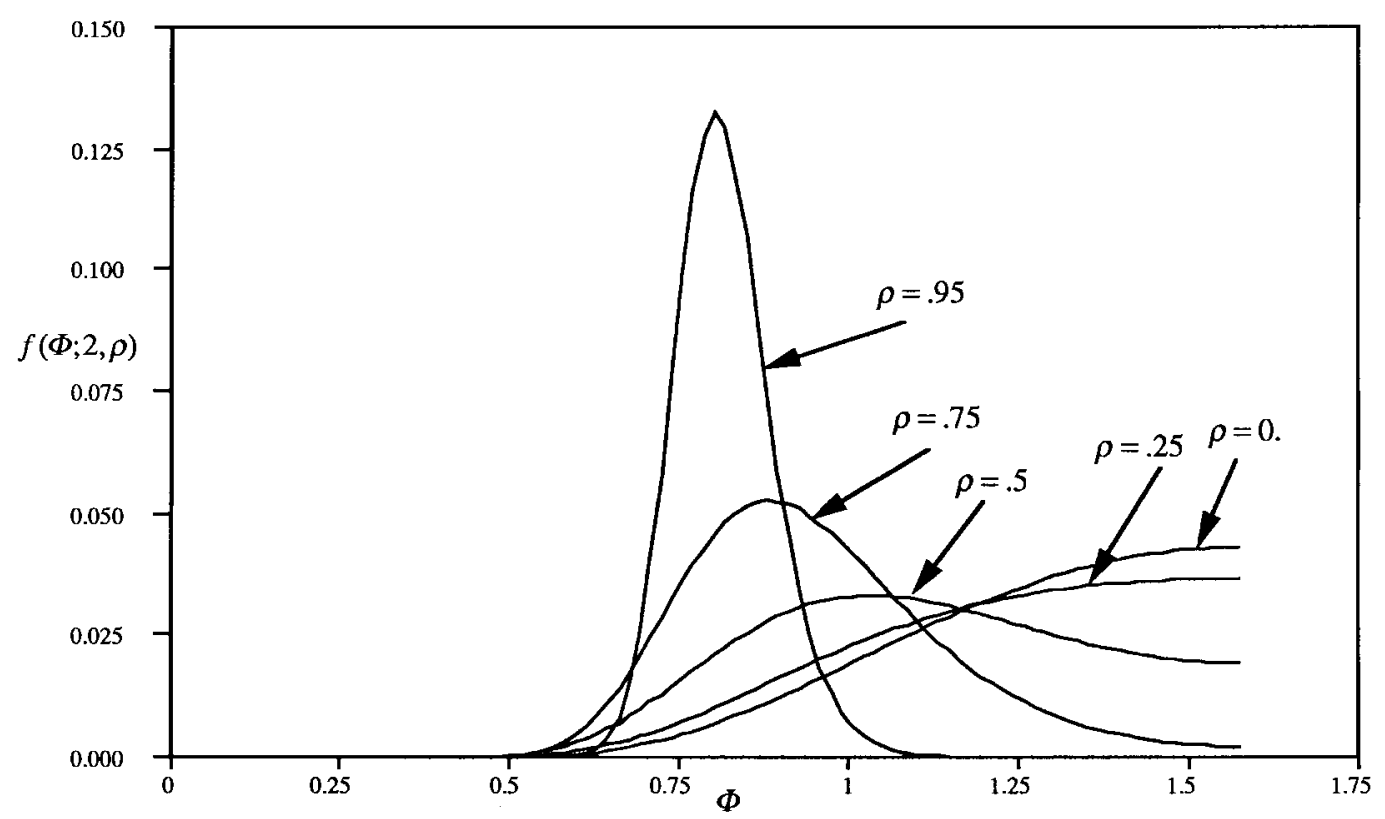

(a)

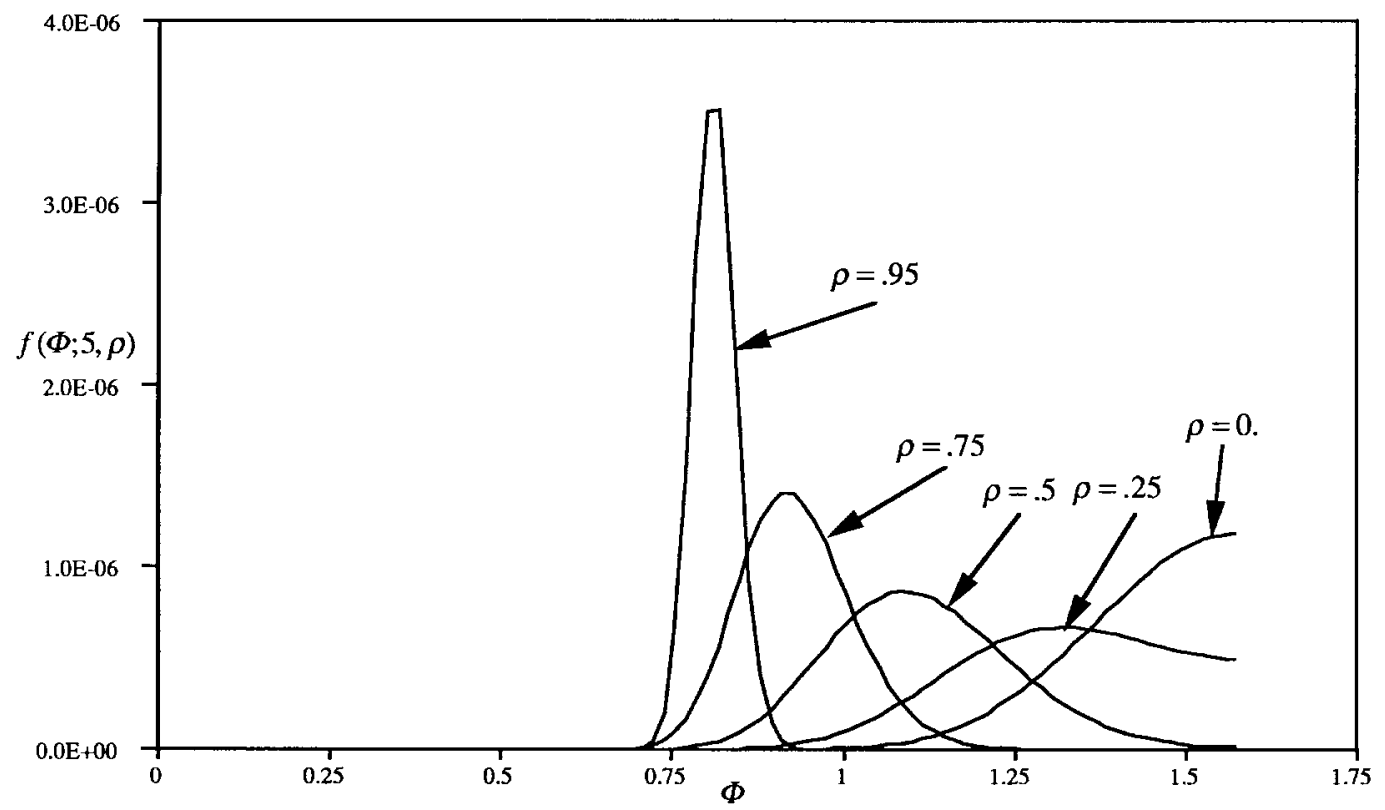

(b)

Fig. 4. The integrand of the parametric Gaussian probability integral (a) $x=2$ and (b) $x=5$.

An interesting relation occurs if we specialize the result in (70) to the BPSK case. Letting $M=2$ in (70), we get

$$
\begin{aligned}
P\left(E ; \phi_{u}\right)= & \frac{1}{2 \pi} \int_{0}^{\pi / 2-\phi_{s}} \frac{\sqrt{1-\rho^{2}}}{1-\rho \sin 2 \Phi} \\
& \cdot \exp \left\{-\frac{E_{s}}{N_{0}}\left(\frac{1}{1-\rho^{2}}\right) \frac{[1-\rho \sin 2 \Phi]}{\sin ^{2} \Phi}\right\} d \Phi \\
& +\frac{1}{2 \pi} \int_{0}^{\pi / 2+\phi_{s}} \frac{\sqrt{1-\rho^{2}}}{1+\rho \sin 2 \Phi} \\
& \cdot \exp \left\{-\frac{E_{s}}{N_{0}}\left(\frac{1}{1-\rho^{2}}\right) \frac{[1+\rho \sin 2 \Phi]}{\sin ^{2} \Phi}\right\} d \Phi
\end{aligned}
$$

where we have further noted that $\cos ^{2} \phi_{s}=1 /\left(1+\rho^{2}\right)$. Since the first integral in the region $\pi / 2-\phi_{s} \leq \Phi \leq \pi / 2$ and the second integral in the region $\pi / 2 \leq \Phi \leq \pi / 2+\phi_{s}$ cancel, then (71) simplifies to the desired result

$$
\begin{aligned}
P\left(E ; \phi_{u}\right)= & \frac{1}{2 \pi} \int_{0}^{\pi / 2} \frac{\sqrt{1-\rho^{2}}}{1-\rho \sin 2 \Phi} \\
& \cdot \exp \left\{-\frac{E_{s}}{N_{0}}\left(\frac{1}{1-\rho^{2}}\right) \frac{[1-\rho \sin 2 \Phi]}{\sin ^{2} \Phi}\right\} d \Phi \\
& +\frac{1}{2 \pi} \int_{0}^{\pi / 2} \frac{\sqrt{1-\rho^{2}}}{1+\rho \sin 2 \Phi} \\
& \cdot \exp \left\{-\frac{E_{s}}{N_{0}}\left(\frac{1}{1-\rho^{2}}\right) \frac{[1+\rho \sin 2 \Phi]}{\sin ^{2} \Phi}\right\} d \Phi .
\end{aligned}
$$

Since the quadrature signal and noise components $\bar{Y}$ and $N_{Y}$ have no effect on the detection of BPSK (note that this is true 
even if $N_{Y}$ and $N_{X}$ are correlated), then the average error probability is also given by

$$
P(E)=Q\left(\sqrt{\frac{2 E_{s}}{N_{0}}}\right)
$$

independent of $\phi_{u}$ or, equivalently, $\rho$. Hence, equating (72) and (73), we arrive at a parametric (in terms of $\rho$ ) expression for the Gaussian probability integral which is a generalization of (2), namely

$$
\begin{aligned}
Q(x)= & \frac{1}{2 \pi} \int_{0}^{\pi / 2} \frac{\sqrt{1-\rho^{2}}}{1-\rho \sin 2 \Phi} \\
& \cdot \exp \left\{-\frac{x^{2}}{2}\left(\frac{1}{1-\rho^{2}}\right) \frac{[1-\rho \sin 2 \Phi]}{\sin ^{2} \Phi}\right\} d \Phi \\
& +\frac{1}{2 \pi} \int_{0}^{\pi / 2} \frac{\sqrt{1-\rho^{2}}}{1+\rho \sin 2 \Phi} \\
& \cdot \exp \left\{-\frac{x^{2}}{2}\left(\frac{1}{1-\rho^{2}}\right) \frac{[1+\rho \sin 2 \Phi]}{\sin ^{2} \Phi}\right\} d \Phi \\
\triangleq & \int_{0}^{\pi / 2} f(\Phi ; x, \rho) d \Phi .
\end{aligned}
$$

Fig. 4 is an illustration of $f(\Phi ; x, \rho)$ versus $\Phi$ for two values of $x$ and values of $\rho$ in the range $0-1$. Note that for $\rho=0$ the function is monotonically increasing in $\Phi$. As $\rho$ increases away from zero, the function $f(\Phi ; x, \rho)$ exhibits a peak and eventually approaches a narrow distribution in the neighborhood of $\Phi=\pi / 4$ as $\rho$ approaches unity. For fixed $x$, however, the area under the various curves for different values of $\rho$ is constant and depends only on the value of $x$ in accordance with (74). As such, the value of $\rho$ can be used to influence the accuracy of the integral evaluation. To illustrate this point, Table I shows the evaluation of the integral in (74) as a function of $\rho$ using a simple Riemann sum of $N$ points for the same two values of $x$ as in Fig. 4 and several values of $N$. Also shown are the corresponding exact values of $Q(x)$ as determined from standard mathematical tables.

\section{The Two-Dimensional Gaussian PROBABILITY INTEGRAL - A NEW FORM}

The normalized (unit variance) two-dimensional Gaussian probability integral is defined by

$$
\begin{aligned}
Q\left(x_{1}, y_{1} ; \rho\right)= & \frac{1}{2 \pi \sqrt{1-\rho^{2}}} \int_{x_{1}}^{\infty} \int_{y_{1}}^{\infty} \\
& \cdot \exp \left\{-\frac{x^{2}+y^{2}-2 \rho x y}{2\left(1-\rho^{2}\right)}\right\} d x d y .
\end{aligned}
$$

Rewriting (75) as in (76), shown at the bottom of the page, we see that we can interpret this integral as the probability

\begin{tabular}{|c|c|c|c|c|}
\hline \multicolumn{5}{|l|}{$x=2$} \\
\hline & \multicolumn{2}{|c|}{$N=100$} & \multicolumn{2}{|c|}{$N=500$} \\
\hline$\rho$ & $Q(x)(E q .(74))$ & $Q(x)$ (exact) & $Q(x)(\mathrm{Eq} .(74))$ & $Q(x)$ (exact \\
\hline 0.0 & $0.2309 \mathrm{E}-01$ & $0.2275 \mathrm{E}-01$ & $0.2282 \mathrm{E}-01$ & $0.2275 \mathrm{E}-01$ \\
\hline 0.1 & $0.2308 \mathrm{E}-01$ & $0.2275 E-01$ & $0.2282 E-01$ & $0.2275 \mathrm{E}-01$ \\
\hline 0.2 & $0.2306 \mathrm{E}-01$ & $0.2275 \mathrm{E}-01$ & $0.2281 \mathrm{E}-01$ & $0.2275 \mathrm{E}-01$ \\
\hline 0.3 & $0.2301 \mathrm{E}-01$ & $0.2275 \mathrm{E}-01$ & $0.2280 E-01$ & $0.2275 \mathrm{E}-01$ \\
\hline 0.4 & $0.2296 \mathrm{E}-01$ & $0.2275 \mathrm{E}-01$ & $0.2279 \mathrm{E}-01$ & $0.2275 \mathrm{E}-01$ \\
\hline 0.5 & $0.2290 \mathrm{E}-01$ & $0.2275 \mathrm{E}-01$ & $0.2278 \mathrm{E}-01$ & $0.2275 \mathrm{E}-01$ \\
\hline 0.6 & $0.2284 \mathrm{E}-01$ & $0.2275 E-01$ & $0.2277 \mathrm{E}-01$ & $0.2275 \mathrm{E}-01$ \\
\hline 0.7 & $0.2279 \mathrm{E}-01$ & $0.2275 \mathrm{E}-01$ & $0.2276 \mathrm{E}-01$ & $0.2275 \mathrm{E}-01$ \\
\hline 0.8 & $0.2276 \mathrm{E}-01$ & $0.2275 \mathrm{E}-01$ & $0.2275 \mathrm{E}-01$ & $0.2275 \mathrm{E}-01$ \\
\hline 0.9 & $0.2275 \mathrm{E}-01$ & $0.2275 \mathrm{E}-01$ & $0.2275 \mathrm{E}-01$ & $0.2275 \mathrm{E}-01$ \\
\hline \multicolumn{5}{|l|}{$x=5$} \\
\hline & \multicolumn{2}{|c|}{$N=100$} & \multicolumn{2}{|c|}{$N=500$} \\
\hline$\rho$ & $Q(x)(\mathrm{Eq} .(74))$ & $Q(x)$ (exact) & $Q(x)(\mathrm{Eq} \cdot(74))$ & $Q(x)$ (exact) \\
\hline 0.0 & $0.2960 \mathrm{E}-06$ & $0.2867 \mathrm{E}-06$ & $0.2885 \mathrm{E}-06$ & $0.2867 \mathrm{E}-06$ \\
\hline 0.1 & $0.2948 \mathrm{E}-06$ & $0.2867 \mathrm{E}-06$ & $0.2883 \mathrm{E}-06$ & $0.2867 \mathrm{E}-06$ \\
\hline 0.2 & $0.2921 \mathrm{E}-06$ & $0.2867 \mathrm{E}-06$ & $0.2877 \mathrm{E}-06$ & $0.2867 \mathrm{E}-06$ \\
\hline 0.3 & $0.2892 \mathrm{E}-06$ & $0.2867 \mathrm{E}-06$ & $0.2872 \mathrm{E}-06$ & $0.2867 \mathrm{E}-06$ \\
\hline 0.4 & $0.2874 \mathrm{E}-06$ & $0.2867 \mathrm{E}-06$ & $0.2868 \mathrm{E}-06$ & $0.2867 \mathrm{E}-06$ \\
\hline 0.5 & $0.2868 \mathrm{E}-06$ & $0.2867 \mathrm{E}-06$ & $0.2867 \mathrm{E}-06$ & $0.2867 \mathrm{E}-06$ \\
\hline 0.6 & $0.2867 \mathrm{E}-06$ & $0.2867 \mathrm{E}-06$ & $0.2867 \mathrm{E}-06$ & $0.2867 \mathrm{E}-06$ \\
\hline 0.7 & $0.2867 \mathrm{E}-06$ & $0.2867 \mathrm{E}-06$ & $0.2867 \mathrm{E}-06$ & $0.2867 \mathrm{E}-06$ \\
\hline 0.8 & $0.2867 \mathrm{E}-06$ & $0.2867 \mathrm{E}-06$ & $0.2867 \mathrm{E}-06$ & $0.2867 \mathrm{E}-06$ \\
\hline 0.9 & $0.2867 \mathrm{E}-06$ & $0.2867 \mathrm{E}-06$ & $0.2867 \mathrm{E}-06$ & $0.2867 \mathrm{E}-06$ \\
\hline
\end{tabular}
that a signal vector $\mathbf{s}=\left(-x_{1},-y_{1}\right)$ received in correlated unit variance Gaussian noise falls in the upper right quadrant of the $(x, y)$ plane. Defining

$$
\bar{S}=\sqrt{x_{1}^{2}+y_{1}^{2}}, \quad \phi_{s}=\tan ^{-1} \frac{y_{1}}{x_{1}}
$$

TABLE I

EVALUATION OF $Q(x)$ By (74)

then using a geometry analogous to Fig. 3, it is straightforward to show that $Q\left(x_{1}, y_{1} ; \rho\right)$ can be expressed as

$$
\begin{aligned}
Q\left(x_{1}, y_{1} ; \rho\right)= & \frac{1}{2 \pi} \int_{0}^{\pi / 2-\phi_{s}} \frac{\sqrt{1-\rho^{2}}}{1-\rho \sin 2 \theta} \\
& \cdot \exp \left\{-\frac{\bar{S}^{2}}{2} \frac{1-\rho \sin 2 \theta}{\left(1-\rho^{2}\right)} \frac{\cos ^{2} \phi_{s}}{\sin ^{2} \theta}\right\} d \theta \\
& +\frac{1}{2 \pi} \int_{0}^{\phi_{s}} \frac{\sqrt{1-\rho^{2}}}{1-\rho \sin 2 \theta} \\
& \cdot \exp \left\{-\frac{\overline{S^{2}}}{2} \frac{1-\rho \sin 2 \theta}{\left(1-\rho^{2}\right)} \frac{\sin ^{2} \phi_{s}}{\sin ^{2} \theta}\right\} d \theta
\end{aligned}
$$

which, using (78a), simplifies still further to

$$
\begin{aligned}
Q\left(x_{1}, y_{1} ; \rho\right)= & \frac{1}{2 \pi} \int_{0}^{\pi / 2-\tan ^{-1}\left(y_{1} / x_{1}\right)} \frac{\sqrt{1-\rho^{2}}}{1-\rho \sin 2 \theta} \\
& \cdot \exp \left\{-\frac{x_{1}^{2}}{2} \frac{1-\rho \sin 2 \theta}{\left(1-\rho^{2}\right) \sin ^{2} \theta}\right\} d \theta \\
& +\frac{1}{2 \pi} \int_{0}^{\tan ^{-1}\left(y_{1} / x_{1}\right)} \frac{\sqrt{1-\rho^{2}}}{1-\rho \sin 2 \theta} \\
& \cdot \exp \left\{-\frac{y_{1}^{2}}{2} \frac{1-\rho \sin 2 \theta}{\left(1-\rho^{2}\right) \sin ^{2} \theta}\right\} d \theta .
\end{aligned}
$$

$$
Q\left(x_{1}, y_{1} ; \rho\right)=\frac{1}{2 \pi \sqrt{1-\rho^{2}}} \int_{0}^{\infty} \int_{0}^{\infty} \exp \left\{-\frac{\left(x+x_{1}\right)^{2}+\left(y+y_{1}\right)^{2}-2 \rho\left(x+x_{1}\right)\left(y+y_{1}\right)}{2\left(1-\rho^{2}\right)}\right\} d x d y
$$


For the special case of $\rho=0$, (78b) simplifies to

$$
\begin{aligned}
Q\left(x_{1}, y_{1} ; 0\right)= & Q\left(x_{1}\right) Q\left(y_{1}\right) \\
= & \frac{1}{2 \pi} \int_{0}^{\pi / 2-\tan ^{-1}\left(y_{1} / x_{1}\right)} \exp \left\{-\frac{x_{1}^{2}}{2 \sin ^{2} \theta}\right\} d \theta \\
& +\frac{1}{2 \pi} \int_{0}^{\tan ^{-1}\left(y_{1} / x_{1}\right)} \exp \left\{-\frac{y_{1}^{2}}{2 \sin ^{2} \theta}\right\} d \theta .
\end{aligned}
$$

In addition, when $x_{1}=y_{1}=x$, we have

$$
Q(x, x ; 0)=Q^{2}(x)=\frac{1}{\pi} \int_{0}^{\pi / 4} \exp \left\{-\frac{x^{2}}{2 \sin ^{2} \theta}\right\} d \theta .
$$

Comparing (80) with (2) we see that to compute the square of the one-dimensional Gaussian probability integral, one integrates the same integrand but only over the first half of the domain. The relation in (80) can also be directly obtained from comparing the symbol-error probability for quadrature PSK (QPSK), namely [5, eq. (4.132)]

$$
P(E)=2 Q\left(\sqrt{\frac{E_{s}}{N_{0}}}\right)-Q^{2}\left(\sqrt{\frac{E_{s}}{N_{0}}}\right)
$$

with the general expression obtained for the symbol-error probability of MPSK using Craig's method [5, eq. (3.119)], namely

$$
P(E)=\frac{1}{\pi} \int_{0}^{(M-1) \pi / M} \exp \left\{-\frac{E_{s} \sin ^{2} \frac{\pi}{M}}{N_{0} \sin ^{2} \theta}\right\} d \theta .
$$

Letting $M=4$ in (82) and equating with (81) gives, after simplification, the equivalent of (80).

\section{REFERENCES}

[1] J. W. Craig, "A new, simple and exact result for calculating the probability of error for two-dimensional signal constellations," in IEEE MILCOM'91 Conf. Rec., Boston, MA, 1991, pp. 25.5.1-25.5.5.

[2] W. C. Lindsey and M. K. Simon, Telecommunication Systems Engineering. Englewood Cliffs, NJ: Prentice-Hall, 1973.

[3] D. Divsalar and M. K. Simon, "Trellis coded modulation for 4800-9600 bits/s transmission over a fading mobile satellite channel," IEEE $J$. Select. Areas Commun., vol. SAC-5, pp. 162-175, Feb. 1987.

[4] _ "Combined trellis coding with asymmetric MPSK modulation," Jet Propulsion Lab., Cal. Inst. Tech., Pasadena, CA, JPL Publication 85-24, MSAT-X Rep. 109, May 1, 1985.

[5] M. K. Simon, S. M. Hinedi, and W. C. Lindsey, Digital Communication Techniques: Signal Design and Detection. Englewood Cliffs, NJ: Prentice-Hall, 1995.

[6] D. Middleton, An Introduction to Statistical Communication Theory. New York: McGraw-Hill, 1960.

[7] C. Tellambura, "Evaluation of the exact union bound for trellis-coded modulations over fading channels," IEEE Trans. Commun., vol. 44, pp. 1693-1699, Dec. 1996.
[8] E. K. Hall and S. G. Wilson, "Design and analysis of turbo codes on Rayleigh fading channels," IEEE J. Select. Areas Commun., to be published.

[9] A. J. Viterbi and J. K. Omura, Principles of Digital Communication and Coding. New York: McGraw-Hill, 1979.

[10] I. S. Gradshteyn and I. M. Ryzhik, Table of Integrals, Series, and Products. New York: Academic, 1980

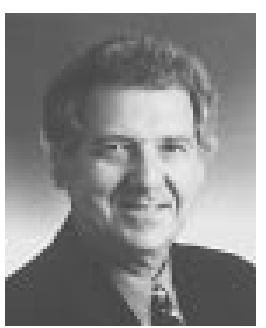

Marvin K. Simon (S'69-M'66-SM'75-F'78) is currently a Senior Research Engineer with the Jet Propulsion Laboratory, California Institute of Technology, Pasadena, CA, where for the last 29 years he has performed research as applied to the design of NASA's deep-space and near-earth missions, which has resulted in the issuance of nine patents and 21 NASA Technical Briefs. Prior to 1998 he also held a joint appointment with the Electrical Engineering Department, California Institute of Technology, where for six years he was responsible for teaching graduate-level courses on random processes and digital communications. $\mathrm{He}$ is known as an internationally acclaimed authority on digital communications with particular emphasis on modulation and demodulation, synchronization techniques for space, satellite, and radio communications, trellis-coded modulation, spread spectrum and multiple access communications, and communication over fading channels. He has published more than 120 papers on the above subjects and is the coauthor of several textbooks. His work has also appeared in the textbook Deep Space Telecommunications Systems Engineering (New York: Plenum, 1984) and he is co-author of a chapter entitled "Spread Spectrum Communications," which has appeared in several handbooks. He is currently preparing a text dealing with a unified approach to the performance analysis of digital communication over generalized fading channels.

Dr. Simon is the co-recipient of the 1988 Prize Paper Award in Communications of the IEEE TRansactions on Vehicular Technology for his work on trellis-coded differential detection systems. He is a Fellow of the IAE, the winner of a NASA Exceptional Service Medal and a NASA Exceptional Engineering Achievement Medal, and, most recently, the winner of the IEEE Edwin H. Armstrong Achievement Award, all in recognition of outstanding contributions to the field of digital communications and leadership in advancing this discipline.

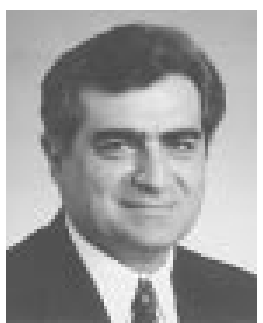

Dariush Divsalar (S'76-M'78-SM'90-F'97) received the Ph.D. degree from the University of California, Los Angeles (UCLA), in 1978.

Since 1978 he has been with the Jet Propulsion Laboratory, California Institute of Technology, Pasadena, CA, working on developing state-of-theart technology for advanced deep space communications systems for NASA space exploration. During the last ten years, he has taught digital communications and coding at UCLA, and recently at the California Institute of Technology. He has published more than 100 papers, coauthored An Introduction to Trellis Coded Modulation with Applications (New York: MacMillan, 1991), and holds six U.S. patents on the above areas. He served as Editor and Area Editor in Coding and Communication Theory for the IEEE TRANSACTIONS ON COMMUNICATIONS from 1989 to 1996.

Dr. Divsalar is the co-recipient of the 1986 Prize Paper Award in Communications for the IEEE Transactions on Vehicular Technology. In 1996 he received the NASA Exceptional Engineering Achievement Medal. 